\title{
NORMATIVE BESTREBUNGEN IM ALTUNGARISCHEN IM SPIEGEL DER BEIGEORDNETEN KONSTRUKTIONEN
}

\author{
ZSUZSANNA PAPP
}

Gewidmet dem Gedenken an Professor Endre Rácz

\begin{abstract}
Auszug
Von einer aus dem Geflecht der Mundarten durch deren Angleichung aneinander entstehenden und sich über sie erhebenden Sprachvariante kann in dieser Zeit im Ungarischen noch nicht gesprochen werden. Vorliegende Studie stellt aufgrund neuerarbeiteten Materials der Kodexliteratur (Übersetzungsliteratur) aus dem Bereich der beigeordneten Konstruktionen - im Spiegel des Vergleichs mit den lateinischen Textvorlagen - innerhalb eines synchronen Segments die Anfangsschritte der Herausbildung und Entwicklung einer sprachlichen Norm dar. Sie versucht, im Sprachgebrauch der damaligen Zeit die historischen Bewegungen nachzuvollziehen, wobei auch auf die Gründe für die Veränderungen hingewiesen wird.
\end{abstract}

Die Herausbildung der Literatursprache ist ein langdauernder Prozess, der in jeder Sprache unter verschiedenen Umständen und zeitlich unterschiedlichen politischen und kulturellen Bedingungen abläuft.

Im Ungarischen hat sich die über den Mundarten stehende Sprachvariante aus dem Geflecht und der Angleichung der Mundarten herausgestaltet; aber im ersten Drittel des 16. Jahrhunderts (der hier behandelten Periode) kann noch keineswegs von dieser normativen Sprachvariante gesprochen werden — um so mehr aber von unterschiedlichen Stufen der Vereinheitlichung, von einer ganzen Reihe von Übergangszuständen, die in zuweilen sehr hochgradiger Bewusstheit der Sprachbenutzer geschaffen werden.

In diesen Prozess fügen sich frühe (aus dem 11-13. Jh.) kleine Textdenkmäler ein, die mit Spuren von entwickelter Textstruktur und stilistischen Fähigkeiten die vorangegangene Entwicklung ahnen lassen: Leichenrede und Bittgebet, Altungarische Marienklage, Königsberger Fragment und Streifen sowie Karlsburger Zeilen.

Diese Sprachdenkmäler werden nicht die einzigen schriftlichen Materiale ihrer Zeit gewesen sein, und auch die Periode zwischen den arpadenzeitlichen Textdenkmälern und dem Beginn des ungarischsprachigen Schrifttums in 
Buchform am Ende des 14. und zu Beginn des 15. Jahrhunderts (Jókai-Kodex, Hussitenbibel usw.) bzw. dessen Ausbreitung im ersten Drittel des 16. Jahrhunderts kann keineswegs ohne Texte gewesen sein (es ist mit verschollenen und verlorengegangenen Sprachdenkmälern zu rechnen). Das beweist die reiche Ausdrucksfähigkeit der Sprache der hier behandelten Periode sowohl im Bereich der Satzkonstruktion als auch in dem ausgefeilten und konsolidierten spezifischen Kodexschreiberstil (-ideal).

Loránd Benkő ist der Meinung, ,... die in den ungarischsprachigen Kodizes des 15-16. Jahrhunderts fixierte und erhaltene ungarische Kirchensprache baut auf der Sprache aus der Zeit unserer Sprachdenkmäler [d. h. der kleinen Textdenkmäler der Árpádenzeit] auf" (Benkő 1980, 354, 362-81; des weiteren Horváth 1944, 80-101; 1980, 75-85; Pais 1953, 430-4; Bárczi 1966, 4-5).

Die sprachliche Vereinheitlichung begann sich in der Schriftsprache auszugestalten und zu formen (Benkő 1960, 32-95; Károly 1961, 385-98; Szathmári $1968,17)$.

Nachdem die Ungarn das Christentum angenommen hatten, entstand und stabilisierte sich im Rahmen der Kirche jene gelehrte Priesterschicht, die dazu berufen war, die offizielle Kirchensprache, das Lateinische, zu verbreiten, zum Träger und Diener der Ziele der Kirche zu werden und die kirchliche Bildung und Kultur zu popularisieren. Diese Tätigkeit verlangte von dieser gelehrten Priesterschicht eine Textgestaltung auf höherem Niveau in Wort und Schrift. Sie wurde von der Kirche dazu ausgebildet, bei der Verbreitung kirchlicher Werke in den Mönchsorden als Übersetzer oder Kopisten lateinischer kirchlicher Werke an der Herausbildung und Entwicklung der ungarischsprachigen Literatursprache teilzunehmen (Horváth 1944, 79; 1980, 81; Mezey 1955, 33; Benkő 1980, 352; Pais 1953, 430-4). Die Herausbildung und Entwicklung der nationalen Literatursprache setzte also vor allem und am kraftvollsten im Bereich der religiösen Literatur ein; seit der zweiten Hälfte des 15. Jahrhunderts verbreiteten sich das Kirchenlatein und die lateinische Bildung zunehmend mehr, denn die Massen der Laien sollten für die Kirche gewonnen werden; die Zeit dafür war um so mehr gekommen, als im Laufe des 13. Jahrhunderts auch in Ungarn die neuen Predigerorden nacheinander entstanden waren und sich verbreiteten, die Franziskaner und Dominikaner. Damit wurden Übersetzungen lateinischer religiöser Bücher notwendig, die in dichter Folge als in Klöstern handgeschriebene und von diesen vertriebene ungarischsprachige Kodizes erschienen und deren Zahl seit der Wende des 15-16. Jahrhunderts stürmisch zunahm (Horváth 1944, 260-7; 1980, 81-5; Pais 1953, 434; Bárczi 1966, 5).

Mit den zwischen den Klostergemeinschaften wandernden, gegenseitig ausgetauschten Kodizes, also - um János Horváth zu zitieren - mit der Wande- 
rung der literarischen Materialien, entstand ein gewisses literarisches Leben. (Der Gömöry-Kodex [1516] z. B. ist das Werk von 11 Händen, unter ihnen von Kató Legéndy und Pál Tetemy, Vikarius von Nagyvázsony.) Diese Literatenschicht — zu der Nonnen und Mönche als Kopisten (wie Lea Ráskay und Márta Sövényházi) und Übersetzer (Pál Váci und András Nyújtódy) gehörten, die also diese Literatur schufen - verfügte über einen gewissen gemeinsamen kirchlichen Sprachschatz; ihre „Literaten“-Tätigkeit war auch insoweit kollektiv, als sie ein Verhältnis zwischen Schreiber (Übersetzer, Kopist) und Leser voraussetzte. Die Gemeinschaft von Vermittlern und Rezipienten (Lesern, Hörern) kannte die Erwartungen der anderen Seite, und diese Erwartungen regten literatursprachliche Bestrebungen an (Horváth 1944, 106; Pais 1953, 435; Pusztai 1978, 485-90). Der einzelne Übersetzer oder Kopist betrachtete es als wichtige Aufgabe, um der wirksamen Kommunikation willen den kaum oder gar nicht des Lateinischen mächtigen Lesern oder Hörern möglichst treu die belehrenden, unterweisenden oder andachterweckenden Texte zu interpretieren: Gebete, Psalmen, biblische Stellen, Heiligenlegenden oder beispielsweise Klosterregeln. Einerseits weist die Klosterliteratur die Spuren der Abhängigkeit vom Lateinischen, des Ringens des Kodexschreibers mit dem Lateinischen auf, andererseits zeigt sich trotz - oder manchmal auch aufgrund - dieser Abhängigkeit in ihr auch die ästhetische Verwirklichung grammatischer Konstruktionsformen.

Ein Bild von der sprachlichen Bewusstheit der Sprachbenutzer bekommen wir nicht nur aus den Angaben, sondern auch aufgrund der Formulierungen und Äußerungen der damaligen Schreiber. So betont der Kartäuser-Anonymus, Verfasser des Érdy-Kodex, die Bedeutung der Übersetzung in die Mutterspracheeine sehr wichtige Erkenntnis im ersten Drittel des 16. Jahrhunderts. Noch sprechender aber sind Sylvesters Bemerkungen in seiner Grammatica, die auf das damalige sprachliche Allgemeinbewusstsein hinweisen. Er betrachtet nämlich gewisse Wörter als inkorrekt, als für den literarischen Sprachgebrauch ungeeignet. Im Zusammenhang mit den Ausdrucksmitteln der beigeordneten Syntagmen, den Konjunktionen, nennt er z. B. die Wortverbindung $s$ monda 'und er sagte' und darin die Konjunktion $s$ 'und' „verderbt" (corrupta). Die kopulative Konjunktion $s$ ist in den Kodizes - gemessen an den vielen Vorkommen von és - tatsächlich selten(er) zu belegen. Ebenso sollte man nach Sylvester die Konjunktion penig zugunsten von kedig vermeiden. Sylvesters Beobachtungen können wir entnehmen, dass schon im ersten Drittel des 16. Jahrhunderts das Bedürfnis auf Auswahl der sprachlichen Mittel vorlag, woraus wir wiederum Schlüsse auf die damalige Stufe der Einheitlichkeit der Literatursprache ziehen können (Balázs 1954, 126-9; Horváth 1944, 234-55; Pais 1953, 436, 440). 
„Das Kennenlernen der ungarischen [Kodexliteratur und] Literatursprache verlangt nicht nur eine detaillierte Beschreibung des Mundartenausgleichs, sondern auch die bis ins Einzelne gehende Kartierung des Ringens mit dem Lateinischen (später dem Deutschen), das Herausheben aus dem Zustand der Mischsprachigkeit“ (Pusztai 1978, 388). Zu dieser „Kartographierung“ möge hier der Gang durch einen kleineren Bereich beitragen, d h. die Darstellung solcher Erscheinungen der normativen Bestrebungen, die in den Kreis der lateinischen Area einbezogen werden können. Zuerst werden jene behandelt, die 1. nicht in das Sprachsystem eingebaut wurden, die sich nicht im Sprachgebrauch verbreiteten, und danach 2. die große Zahl der als Produkte der Übersetzungsliteratur vorkommenden Synonymhäufungen, die spezifisch erklärenden Konstruktionen, die Konstruktionsproblematik und die stilistischen Formen ihrer Arten. Diese Konstruktionen konnten entstehen, weil der Übersetzer und Kopist mit dem Lateinischen kämpfte, aber nicht nur mit ihm, sondern auch mit der durch die Übersetzung geschaffenen ungarischen Kirchensprache. Deren Schwerfälligkeit rührte freilich nicht nur von der Übersetzung aus dem Lateinischen her, sondern ergab sich auch daraus, dass der Text der Vorlage auch selbst zur Schrift- und nicht zur gesprochenen Sprache gehörte (Benkô $1980,353)$.

1. Eine jeweils kleine Stufe oder Station im Process der Vereinheitlichung bilden - wie schon ausgeführt - die individuellen Bestrebungen, die das Ringen mit dem lateinischen Text oder das Kopieren eines Textes mit schwerfälliger, verwickelter Satzstruktur hervorrief. Der Einfluss und die Rolle des Individuums auf die und bei den sprachlichen Veränderungen ist nicht zu bestreiten. Aber von den Verwirklichungen seiner sprachlichen Bewusstheit bleiben nur einige erhalten oder werden zu Präliminarien bzw. Ausgangspunkten wieder neuer Sprachvarianten, andere widersetzen sich - aufgrund ihrer Isoliertheit oder aus anderen Gründen - der Veränderung, haben keine Folgen, d. h. sie gelangen nicht ins Sprachsystem. (Die hiesigen Angaben stammen aus dem Minimalkorpus der Vorarbeiten für A magyar nyelv történeti nyelvtana, Bd. I. 1992, II/2 1995, bzw. aus individueller Sammlung und Zufallsfunden in durchgesehenen Texten.)

1.1. Als Beispiele für die genannte Erscheinung sind die in der Hussitenbibel (Wiener und Münchner Kodex) konsequent und bewusst verwendeten Konjunktionen zu nennen, die erklärende és mert 'etenim' und die konsekutiven és úgy, és igy 'itaque', mit der Einschränkung, dass diese Lehnübersetzungen noch nicht unbedingt auf normative Absicht zurückgehen, sondern nicht zu un- 
terschätzende Sprachneuerungen, sprachliche Einfälle sind. Beispiele: MünchK. 100ra: „Tu hiutoc èngèmet mefte $2 \mathrm{n}^{\mathrm{c}} z$ v2nac / $\mathrm{z}$ iol mongatoc $z$ me2t [etenim] vagoc"; MünchK. 38ra: „3́ombat èmbėzèke2t lot / nem a3 èmbe2 3ombatert / z ug [Itaque] èmbe $2 \mathrm{n}^{\mathrm{c}}$ fia 3́ombatnac es v2a“; BécsiK. 1: „Mert hallotta vala hog vr tèkèntèttè volna onèpèt $z$ adot volna onèkic ètkèkèt $z$ ig [itaque] ki èzèdè o zazādoklatt'anac hèlèbọl“. Diese Konjunktionen blieben isoliert, ihr begrenzter Gebrauch verhinderte auch, dass sie zu Zusammensetzungen wurden, selbst wenn sie sich - wie aus den Angaben zu sehen ist - funktional als Einheit verhielten. Sie gelangten nicht in den Sprachgebrauch, sozialisierten sich nicht, es gab sie nur in den von Tamás Pécsi und Bálint Újlaki übersetzten die Hussitenbibel enthaltenden — Kodizes (Rácz 1963, 26-8; Juhász 1992, 789; Papp 1995, 746, 750; des weiteren Benkô 1988, 58).

1.2. Ebenso arealer Einfluss des Lateinischen ist die Übernahme der Konjunktion nam 'denn' mit genauer und bedeutungsmäßiger Entsprechung durch die unter Konjunktionsmangel leidenden Kodexschreiber. Auch der Gebrauch dieser Konjunktion blieb begrenzt. Eine ganze Reihe von Beispielen lassen sich aus Kodizes beibringen, etwa ApMélt. 19: „Mÿth tazÿgalzh engemeth nam en te zeretod vagÿok"; NádK. 191: „Iôttetồk mikeppen tolvayra, tồồckel, es rudackal megfogni engo̊meth Nam mindo̊n napon veleto̊c voltam a templomba tanetvan ... : Tamquam ad latronem existis cum gladiis et lignis comprehendere me? Cotidie eram apud vos in templo docens ...". (Im lateinischen Original findet sich die Entsprechung des ungarischen nam nicht!) - Interessanterweise stoßen wir auch in einer mittelalterlichen Missilesammlung auf nam in erklärender Konkunktionsfunktion: 1534: ,a germek lowat a fwre bochasd ... ha meg nem etetyk wele a fwwet tehat elwez a lo, nam ('denn') talalz ot eleg retet" (KLev. 127.). - Später allerdings geht sein Gebrauch zurück, bis es schließlich in der Sprache verschwindet (Velcsov 1987-1988, 211-7; 1997, 173-7; des weiteren Juhász 1992, 801-2; Papp 1995, 751).

1.3. Die Sprachbenutzer aller Zeiten verwenden - aus dem Zwang heraus, Neues zum Ausdruck bringen zu müssen - in den meisten Fällen das vorliegende Sprachmaterial höchst einfallsreich, wenn es gilt, logisch kompliziertere inhaltliche Verhältnisse wiederzugeben. Und gerade in der Zeit der Übersetzungsliteratur stand der Kodexschreiber, wenn er Texte spezieller Bestimmung ins Ungarische übersetzte oder die Übersetzungen kopierte, häufig vor der Aufgabe, die langen scholastischen Erörterungen mit komplizierten Satzkonstruktionen durch Erklärungen und Entfaltungen sich selbst oder dem Leser verständlich zu machen, während er doch um die der Mitteilungssituation am besten ent- 
sprechenden Ausdrucksformen bestrebt war (Benkő 1988, 53). Damit war er auch zu eigenen (individuellen!) Veränderungen fähig, wenn er aus dem von ihm beherrschten Sprachmaterial schöpfte. Bei den Ausdrucksmitteln des Verhältnisses beigeordneter Konstruktionsglieder tauchen in Konjunktionsfunktion, sich wie Konjunktionen verhaltend, die Wortformen értsed, értvén, értsen 'verstehe es!' auf. Diese sind Imperativformen der 2. und 3. Pers. Sing. des Verbs ért 'versteht' sowie des suffixes -vén, die in der Wortkonstruktion wie zwischen Gliedsätzen in identifizierender, präzisierender (außerdem verzögernder wie auch Nachdruck gebender) Funktion agieren. Außer den Angaben von Simonyi $(1881,259)$ und NySz. (Szarvas - Simonyi 1890-1893, 685) hat sich ihre Zahl auch beim Suchen in den Kodizes nur um einige weitere erhöht. Es scheint, dass man sie nicht allgemein benutzte, sondern sie eher nur individueller Sprachgebrauch der Schreiber des Tihanyer, des Lobkowitz- und des Érdy-Kodex in den erhebenden, erörternden Teilen der Predigten waren.

Die 2. Pers. Sing. Imp. értsed (von ihr gibt es mehr Angaben als von értsen und értvén) brachte prägnant die nachdrückliche Aufforderung zum Ausdruck, etwas zu verstehen oder sich einzuprägen. Die Bedeutung in den Angaben: 'denn, nämlich, d. h.', ja sogar so etwas wie 'nota bene!'.

Die Angaben dokumentieren die Vorkommen von erklärenden Konstruktionen, für den sonstigen Gebrauch (Nachdruck, Verzögerung) s. Papp (1996, 237-41).

Zwischen zwei Konstruktionsgliedern drückt értsed Darlegung und Präzisierung aus: LobkK. 64: Oth erčhed az pokolba; aber einige Zeilen tiefer, 64: Oth. az az pokolba - also ist der Gebrauch beider Ausdrucksmittel austauschbar. (Dieser Kodex ist besonders reich an Darlegungs-Erklärungskonstruktionen: 87: belöl vaǵon az. az. a lelocbe, 70: akoron tvdnia mint. itelet napian usw.); LobkK. 70-1: „O akoron kellemetôs lefzôn mindôn bekefegel zenvedôth haborvfag. es: az ragalmazoknac mindồn alnokfag. $\stackrel{\mathrm{e}}{\mathrm{v}}$ zaiokat bee dvgia $\mathbf{\uparrow}$ Tahat ['tunc'] erched iteletnec napian vigad es ôrvl mindồn aitatos embör"; aber 65: Akoron Az az iteletnec napian; TihK. 206: „Errôl monǵa ... damalcenus: Az anǵalok [anǵalokrol] erćed az $\stackrel{e}{o} r d \stackrel{e}{o} g o \stackrel{e}{o}$ kro l: mikepen nem uotak: fem uannak: fem leznek az penitencianak el ueuoi i“.

Der Schreiber des Érdy-Kodex benutzt die Form értvén zwischen den Konstruktionsgliedern mit der Bedeutung 'das heißt': 553: „zolgalnak vala ewneky, erthween az wr iftennek".

Sicherlich wird auch eine breitere Materialsammlung in der untersuchten Periode das Bild von értsed und den anderen Formen nicht mehr verändern.

Zur Einsicht in die folgende Periode sei aus Erdélyi Magyar Szótörténeti Tár (Szabó 1982, 377) eine Angabe genannt, in der értsed ein nachgestelltes 
identifizierendes erklärendes Konstruktionsglied verbindet: um 1683: „az Prédikatornak ... ad minden ember ... egy egy öregh veka buzat melly nem csak az Erdelyi hanem az Somlyai es Marghithai vekana is nagyubb, ertsed egy egy vekanal ...".

Das értsed wurde nicht und konnte nicht zur Konjunktion werden, da es nur einem engeren Kreis der Sprachbenutzer geläufig war und sich die häufig gebrauchten sprachlichen Elemente zu grammatikalisieren pflegen; hier also gab es keinen Grund für sprachliche Änderung.

Der andere und wichtigere Umstand, der die sprachliche Änderung verhinderte, ist die Tatsache, dass die 2. Pers. Sing. prägnant den Imperativ kennzeichnete und damit auch die Lautform den am Wortende im übrigen leicht geschehenden Veränderungen widerstand, so etwa der Angleichung, Abschleifung und Reduktion (s. die Entwicklung von 2. Pers. Sing. Imp. hagyd 'lass!' durch Wortartwechsel und Assimilation $g y d>d d$ zum Modalwort, s. weiter die Entstehung der Modalwörter *találom > talám > talán > tán 'vielleicht', látom $>$ lám 'siehe!' sowie das Schicksal von hiszëm > hiszën 'denn'; vgl. noch den suffixlosen Zustand der auf die 3. Pers. Sing. verweisenden Form avagy 'oder'.)

Im folgenden werden Erscheinungen behandelt, die sich verbreiteten und in den Sprachgebrauch eingebaut wurden.

2. Die spezifisch erklärenden Konstruktionen sammelten sich im Laufe der Materialsammlung und Verzettelung beim Vergleich mit dem lateinischen Vorlagetext und ließen sich von den „traditionellen" darlegend erklärenden Syntagmen trennen. (Wichtig hinsichtlich der lateinischen Textvorlage ist, dass der erreichbare und entsprechendste lateinische Text benutzt und als Quellentext von mit betrachtet wurde, wobei möglicherweise nicht dieser dem Übersetzer vorgelegen hat.)

Diese zwei- oder mehrgliedrigen Konstruktionen sind so entstanden, dass der Übersetzer ein lateinisches (seltener anderssprachiges) Wort aus dem vorliegenden Quellentext nicht mit einem Wort, mit der ungarischen Entsprechung, wiedergab, sondern mit zwei oder mehreren, und zwar Synonymen. Bei den Konstruktionen stößt man auch auf solche, in denen irgendein Konstruktionsglied ein in den Klöstern bekannteres (fallweise aber doch nicht genug oder sicher bekanntes) lateinisches Wort ist, weshalb der Übersetzer oder Kopist zu seiner Beruhigung auch die ungarische Entsprechung hinzufügte. Der Kodexschreiber erklärt und glossiert also gemäßs seinem Sprach- und Stilgefühl, seinem Eifer und seinen Kenntnissen, nimmt für seine Arbeit eventuell auch eine andere Übersetzung zu Hilfe, so wie András Nyújtódy, der Übersetzer (von 
gut zwei Dritteln) des Székelyudvarhelyer Kodexes, den Text des Wiener Kodexes zu Rate zog. Da die meisten ungarischen Kodizes (eventuell sogar mehrfache) Kopien sind, mag auch der Kopist entsprechend seines Sprachgefühls und eventuell Dialektes den Text verändert und mitgestaltet haben. Dieser „wichtigtuerische“, „präzisierende" Stil ist charakteristisch für die Kodexschreiber, dies alles ist mit ihrer Neigung zu übertriebener Ausschmückung und ihrem Bestreben nach Feierlichkeit zu erklären. Von Textbeständigkeit kann zu jener Zeit noch nicht gesprochen werden, wobei aber bei den sakralen Texten aus der Bibel die Psalmen und das Neue Testament eine Ausnahme bilden, weil sie streng textgetreu übersetzt wurden. Diese dogmatische Auffassung war dadurch begründet, dass den wichtigsten Teil der Bibel die Evangelien bilden und diese deshalb nicht verändert werden durften (vgl. Nagy 1981, 44). Andere Teile aber, beispielsweise die alttestamentlichen Geschichten, wurden von Kodexschreiber wegen der Übersetzungsschwierigkeiten auch mit eigenen Erklärungen gewürzt. Dies tat auch der schon erwähnte András Nyújtódy, der für seine Schwester, die Nonne Judit, das biblische Buch Judit ,aus dem Lateinischen" übersetzte, wie er in seiner Widmung des Werkes schreibt, und tatsächlich den Originaltext um an Glossierung erinnernde Klammererklärungen bereichert hat.

Wir sehen also, dass der Übersetzer oder Kopist bei seiner Arbeit auf das Problem der grammatischen Unterschiede beider Sprachen stieß. Diese Arbeit ließ ihn die Eigenheiten seiner Muttersprache erkennen und zwang ihn dazu, ihren Reichtum auszuschöpfen: unter den Ausdrucksmöglichkeiten zu wählen und Stellung zu beziehen. So war er dazu gezwungen, gewisse Regeln oder Normen aufzustellen (vgl. Szathmári 1980, 44).

Die hierzu gezählten Konstruktionen zeigen, dass der Kodexschreiber einen alternativen Wortgebrauch pflegt, als suche er die Wörter wörterbuchartig zusammen. János Horváth äußert sich so über die Arbeit der Übersetzer: ,,sie sind keine Textschreiber, sondern nur Erklärer der Wörter des fremden Textes ... nicht ,Schriftsteller', sondern lebende Wörterbücher, und sehen es als ihre Berufung an, sogar mehrere Entsprechungen des lateinischen Wortes anzugeben" (Horváth 1944, 275). Im wesentlichen ist diese Feststellung unbestreitbar, zu bemerken ist aber, dass die Auswahl der Synonyme und die Suche nach den schönsten, meistentsprechenden und dichterischsten, und darüber hinaus ihre steigernde, Erhabenheit ausstrahlende Häufung vielfach nicht den mechanischen Wörterbuchblätterer, sondern einen Textschöpfer zeigt.

Von einigen der ungarischen Kodizes - etwa Nagyszombater Kodex (15121513), Margaretenlegende (1510), Székelyudvarhelyer Kodex (1526-1528), Teleki-Kodex (1525-1531), Birk-Kodex (Rohübersetzung der Regeln von Pál Vá- 
ci, 1474), Nádor-Kodex (1508), Buch der Gleichnisse (Példák könyve) (1510) usw. - lässt sich eine Reihenfolge der Häufigkeit aufstellen, wie gern ihre Übersetzer, Kopisten und Kompilatoren bei ihrer Arbeit die explizierende Detaillierung verwendeten, aber die Entstehung und Häufigkeit dieser Konstruktionen wurden auch von gattungsmäßigen Gründen beeinflusst: vom Thema und der Bestimmung der Texte.

Die Konstruktionsglieder bestehen aus verbalen und nominalen Wortarten. Wichtig sind die Ausdrucksmittel des synonymen Verhältnisses der Konstruktionsglieder: die Konjunktionen és 'und', vagy, avagy 'oder', azaz 'd. h.' oder die konjunktionslose Verbindung bzw. die Kombinierung der Ausdrucksmittel. Meiner Meinung nach kommen die kopulative Konjunktion és und die disjunktiven Konjunktionen vagy, avagy nicht wirklich mit ihrem eigenen Wert und Funktion vor, sondern eher in der darlegenden, präzisierenden oder identifizierenden Rolle von 'mit anderen Worten, d. h.', bezeichnen aber auch die Ergänzung, Detaillierung oder Wählbarkeit. Wenn also die Konstruktionsglieder mit és verbunden sind, dann nicht im kopulativen Verhältnis, und wenn sie mit vagy verbunden sind, dann nicht im Verhältnis der Wahl.

Hinsichtlich seines Bedeutungsinhalts und des Verhältnisses zu den anderen kann jedes Konstruktionsglied das Wort für einen engeren Begriff oder gerade entgegengesetzt eine ausführlichere Beschreibung, ja eine ganze Reihe von Erklärungen sein; ein Glied kann auch als genauere Benennung eines anderen dienen.

2.1. In einer Gruppe der Wortkonstruktionen entsprechen einem (eventuell zwei) lateinischen Wort des Quellentextes im Ungarischen zwei oder mehrere Wörter.

(a) Das kann auch so geschehen, dass eines der Konstruktionsglieder die „Roh-“ Übersetzung des lateinischen Wortes ist: PéldK. 67-8: „erews embereket ... le haytok es le hullatok : vitales spiritus ... reflecto“, 67: „elmeryetewk meg az ty ky meneltewket es halaltokat : Discernetis exitum"; Es gibt auch ein Beispiel (JókK. 136), wo das eine Konstruktonsglied die wortgetreue Übersetzung des lateinischen Wortes ist: „vr yefus cristus meg yelenek ... mendennek

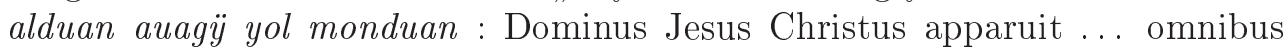
benedicens"; usw.

(b) Mit Synonymenhäufung wird die Ausdrucksweise differenzierter: MargL. 4: epehtenek ragkanak : aedificaverunt, „valtoztateek hozattatek az clastromban : translata est in claustrum“, 12: „,gyan azon fedelet auagy ruhat: dictum velum"; TelK. 24: „az vtakat meg iaria vala: keringi vala : circuibat vias omnes“, 17: „maradekoknac: vnokaknac: auagy o̊ ro ko le knec: gômôlčet nem kồuetec : 
fructum posteritatis consecuti non sunt", 26: „kezde ... zomorogni: es igôn nagon keferögni ko no e uet hullatni : Coepit ... contristari et lacrimari nimis.

Folgende Angabe des Teleki-Kodex ist das vielleicht schönste Beispiel dafür, einen farbigen, erhabenen Stil zu verwirklichen, der die Dinge sichtbar machen und auf die Empfindungen wirken wollte: 18: „,cent anna azzon viragozuan: terömthuen: es gómo̊lčo zuen fogadna : Anna fecundata conceperit" mittels Aufzählung der Entwicklungsstufen gibt der Kodexschreiber die lateinische Konstruktion durch das dichterisch schöne Bild vom blühenden und dann früchtetragenden Baum wieder.

Die steigernde Wirkung der detaillierenden Häufung setzen die Kodexschreiber vor allem bei den Passionszenen ein: NagyszK. 17: „, megmöuetenec keleritenec, es kenzanac: me afflixerunt", ,azén gengelegós zep zerelmes orczamat ... haǵapalaac es pogdôleek : faciem meam amabilem ... conspuebant" 13: „ki ... genéerülego s kedues, es zerelmes : quae ... eft amabilis" usw.

Die detaillierende Beschreibung kann auch so geschehen, dass der Kodexschreiber bei der Übersetzung auch Unterschiede nach Geschlechtern macht: BirkK. 2b: зabad fiak leaniok : liberae; PéldK. 72: ,kyk aleytyak vala magokat vraknak es azzonyoknak lenny : Qui se credebant dominos" — auch dieses Beispiel zeigt, dass es damals möglicherweise noch die Bedeutung asszony 'Herrin, Fürstin' gab.

Mit Verben inhaltsvollerer Bedeutung wird die Beschreibung anschaulicher: NagyszK. 8: „rolayat zedhellec, es vehellek : rosam habeant".

(c) Der alternative Wortgebrauch (Übersetzung) kommt im Birk-Kodex in großer Zahl vor, dessen Rohübersetzung von Pál Váci stammt und Klosterregeln enthält. Da in den dadurch entstandenen Syntagmen die Glieder einander sehr ähnlich sind, fast identische Bedeutung haben, konnte leicht eine ursprünglich trennende Konjunktion, vagy, auf analogen Einfluss des avagy (ähnlicher Funktion) in die Konstruktion gelangen: 3a: „se iriak vag iegeззеk : ne notetur", 4a: „3ent kenetet vige vag vifelie : sacram deferant unctionem“ usw. - Hier ist anzumerken, dass es sich in gewissem Grade um Wortspaltung handelt.

(d) Die Kodexschreiber passten sich in ihrer Arbeit — von Nyújtódy war im Zusammenhang mit der Übersetzung des Székelyudvarhelyer Kodex schon die Rede - insofern der Leser- oder Hörerschaft an, dass sie mit explizierenden Einschüben ein besseres Verständnis förderten: SzékK. 10: ,izrahélnek fyay ... mégh félémenek, (azaz, megh yedenek)" — auch in diesem Fall ist die parallele Textübersetzung sprechend: BécsiK. 15: „.. megfelemen ${ }^{c}$ : filii Israël ... timuerunt".

Zuweilen machte man den Text durch eine ganze Reihe von Erklärungen mit verschiedenen erklärenden Konjunktionen noch verständlicher: SzékK. 35: 
„mynden néép, (azaz mynd az ko zo neneeg) Mynd egeez az eyel az eǵhazban (azaz, az fynagogaban, awaǵ, az g'velekezetben) ymadkozanak : omnis populus, et per totam noctem intra ecclesiam oraverunt".

(e) Eine Wortkonstruktion konnte auch auf die Weise zustande kommen, dass der Übersetzer oder Kopist - ganz sicher — zur „Verstärkung“ Synonyme aufführte: NagyszK. 17: „azén ... orczamat ... haǵapalaac es poegdo seek : faciem meam ... conspuebant". Eventuell — da hagyap(ás) auch 'osculum; Kuss' bedeutete - hat ihn zu diesem Verfahren auch seine gute Muttersprachenkenntnis oder Mundart bewogen.

Ähnliche Umstände werden eine Rolle beim Entstehen der folgenden Wortkonstruktion aus dem Cornides-Kodex (139v) gespielt haben: ,zent praxedis azonnak huga vagy evcche : soror Praxedis". — öcs war ursprünglich ein Wort mit breiterer Bedeutung: 'jüngerer Bruder', 'jüngere Schwester', verengte sich später aber auf den Mannesstamm; s. dazu TESz. öcs.

Die Erscheinungen dieser Gruppe könnten mit dem Terminus explizierende Synonymenhäufung ezeichnet werden.

2.2. Das in den Text eingeflochtene Fremdwort - das zumeist lateinisch ist kann auch mit seiner ungarischen Entsprechung zusammen (mit oder ohne Konjunktion) eine Wortkonstruktion bilden.

(a) Reich an solchen ,zweisprachigen Syntagmen“ sind vor allem Texte belehrenden Zweckes (Ordensregeln), unter anderem der Horvát-Kodex, aber noch mehr die Rohübersetzung des Birk-Kodex mit Verbesserungen, Streichungen und Einschüben. BirkK. 1a: ,oзtaflek feidelm aззоntol 〈азазрrioriflatol〉... elet es ruha : distribuator ... a praeposita vestra victus et tegumentum“ —im Kodex bedeutet das bekanntere lateinische Wort in spitzen Klammern den nachgesetzten Einschub; 2b: „difciplinat fegelmet ørømeft ... vegen on maga : disciplinam libens habeat" — in diesem Falle geschah die Erklärung mit dem ungarischen Wort.

Das an Glossierung erinnernde Verfahren kann am Jókai-Kodex 48 gezeigt werden: „en [con] Cci[enti]am lelkem lemegÿben nem segÿengett meg engemet : in nullo conscientia me reprehendit" — lelkem ist über die verkürzte Form von confcientiam in den Zeilenzwischenraum geschrieben; 111: „ffrater lleo bod [boldog] ferencзnek ... co[n]feltora gÿwuontatoya Ira : frater Leo ... confessor sancti Francisci scripsit", 45: Pater: Attyam : Pater.

Die Konstruktionsweise der in der Klosterliteratur „gängigen“ lateinischen Wörter mit ihren ungarischen Entsprechungen mit (és, vagy, avagy, azaz) oder ohne Konjunktionen kann mit noch weiteren Angaben belegt werden: GuaryK. 
30: „eǵiǵú lelkot es conscienciat meg habozeÿtod : simplices conscientias turbas".

Ein lateinischer Eigenname bildet mit seiner ungarischen Entsprechung (ungarischem geographischen Namen) eine Konstruktion: NagyszK. 15-6: „az olyuetim heǵere, az az olayfanak heǵere : in montem oliveti".

Unter den um wirkungsvolle Kommunikation bemühten Kodexschreibern ragt Lea Ráskay, eine Nonne von der Haseninsel, durch ihre entwickelte Schriftkultur und gutes Stilempfinden hervor. Aus den von ihr kopierten Kodizes seien hier Beispiele der Margaretenlegende genannt, die ihre Biographie und einen ordensgeschichtlichen Rahmen enthält: 9: „az silenciomot az vezteksegtartast hatra nem vety vala : silentio ... non proiecto“, 11: „meg oluastagÿa vala az passiot cristusnak kennüat : passionem Christi“.

Interessant ist die Übersetzung von velum, ebenfalls in der Margaretenlegende: 16: ,az ev veloma auagÿ kezkenevye [velum suum] ... annera vizesevl vala meg. hog annak vtanna ez zent zyz . az velomot auagÿ kezkenevt [velum suum $]$ meg facharÿa vala . es az sÿralmnak vize kÿ ju vala belevle az velombol". Anderswo in diesem Kodex - dies wurde bereits zitiert - gibt der Kodexschreiber velum mittels zweier ungarischer Wörter wieder: 12: fedelet auagy ruhat.

(b) Außer den Erklärungen lateinischer Wörter gibt es - im untersuchten Korpus - auch die Angabe eines deutschen Lehnwortes, das der Kodexschreiber ebenfalls mit Erklärungen versehen zu müssen glaubte: VitkK. 64: „Se valakit toaba meg ne strofol' [nec aliquem de cetero reprehendas], vag meg ne feǵelmeź, hanem ha nuiluan istennec ellene vetkezic“. „Mit gewisser Unsicherheit kann auch darauf geschlossen werden, dass der kopierte Text selbst von einem Übersetzer fremder Zunge stammte, weil nur für einen solchen die Übersetzung akzeptabel war" - meint István Pusztai im Vorwort zum VitkovicsKodex (Pusztai 1991, 20).

Die hier (in Punkt 2.2.a und b) gezeigten Verfahren der Übersetzer- und Kopierarbeit - ob also zu einem lateinischen oder anderssprachigen Wort die ungarische Entsprechung hinzutritt oder die Erklärung und Verständlichmachung umgekehrt geschieht - könnten mit dem Terminus explizierende Glossierung charakterisiert werden.

3. Im Zusammenhang mit den Erscheinungen, die die normativen Bestrebungen zeigen, stößt man auf einen umstrittenen deskriptiven grammatischen Problemkreis, einen Übergangsstreifen zwischen Bei- und Unterordnung, über den unbedingt kurz zu sprechen ist. 
In der Fachliteratur wird der betreffende Konstruktionstyp - wenn auch mit vielen Ausnahmen - zu den Appositionen gerechnet und zudem zu deren identifizierendem Typ.

Hier wäre es zu lang, und es ist auch nicht mein Ziel, die seit Jahrzehnten zu verfolgende deskriptive Grammatikdiskussion wiederzugeben, deshalb möchte ich nur einige wichtigere, das Thema betreffende Knotenpunkte hervorheben. Dabei stütze ich mich auf die gründliche, zusammenfassende und analytische Studie von Judit Balogh-Kardos über die beigeordneten Wortkonstruktionen und ihre Grenzfälle (Kardosné 1989, 57-90), wenn ich über die enger zu diesem Themenkreis gehörenden Meinungen über die Apposition und besonders die identifizierende Apposition berichte (ebd. 68-72).

3.1. Für ein beigeordnetes Syntagma hält Dénes Szabó die Apposition in seiner deskriptiven Untersuchung $(1958,281-5)$ ), wobei er die bezeichneten (mit Konjunktionen) und unbezeichneten (konjunktionslosen) Konstruktionen unterscheidet.

Gleichfalls zum Kreis der Beiordnungen rechnet László Antal (1964, 61-8) die Apposition und erklärt, dass „die traditionelle ungarische Grammatik ... einen ihrer größten Irrtümer begangen hat, als sie die sogenannte Apposition oder das nachgestellte Attribut - als Attribut qualifizierte".

Andere, wie József Tompa (1962a, 261-2), betrachten die Apposition als Übergang zwischen Bei- und Unterordnung, ähnlich urteilt auch Jolán Berrár $(1957,124)$, aber in einer späteren Arbeit $(1967,453)$ behandelt sie die Apposition bei den Attributen.

In der genannten Arbeit würde Balogh-Kardos die darlegenden erklärenden Konstruktionen (auch die mit Konjunktion konstruierten) mit der identifizierenden Apposition in eine Gruppe einordnen und die mit und ohne Konjunktion erfolgende Identifizierung voneinander unterscheiden; sie führt die Meinung von Vilmos Farkas $(1962,326)$ an, der den Übergangscharakter des genannten Typs ebenfalls spürt und von spezifischer Identifizierung spricht.

1977 setzte die Diskussion zwischen István Jakab und Sándor Károly im Magyar Nyelvốr ein. Jakab brach eine Lanze für die Beiordnung der Apposition, indem er auch auf die auftauchenden systematischen Probleme hinwies (Jakab 1977, 9-19; 1978, 293-9), Károly betrachtet in seinem Antwortartikel die Apposition als Unterordnung (Károly 1978, 46-50). In einer früheren Arbeit (1958) erörtert er außer den attributiven Appositionen und der Apposition im Kapitel „Die spezifische identifizierende Apposition“ noch die das sog. unmittelbare homogene Verhältnis darlegenden Konstruktionen, die spezifisch identifizierenden Konstruktionen. Deren Glieder gehören zu den verbalen (partizipialen), adver- 
bialen, adjektivischen und numeralen Wortarten. $\mathrm{Zu}$ den verbalen bemerkt er mit Nachdruck, sie seien ein sehr seltener Typ (1958, 45-6). Bei ihren Untersuchungen am historischen Material zu den Arbeiten an der historischen Grammatik der ungarischen Sprache reiht Antónia S. Hámori (1991, 706-11; 1995, 396, 404) im Zusammenhang mit dem Konjunktionsgebrauch der appositionellen Konstruktionen in altungarischer Zeit den hier behandelten Konstruktionstyp bei den Appositionen ein und spricht der Konjunktion hervorhebende, Nachdruck gebende Funktion zu; dabei betrachtet sie solche Konstruktionen, zwischen deren Gliedern die ,für das beigeordnete Verhältnis typische kopulative Konjunktion steht, aber in nicht kopulativer, sondern steigernder, Nachdruck gebender Funktion" (1981, 151), als Kontamination des identifizierend appositionellen und des beigeordneten, kopulativen Typs.

Meiner Meinung nach ergibt sich aus dem Übersetzungscharakter der ungarischen Klosterliteratur, dass diese aus pragmatischen und stilistischen Gründen entstandenen Konstruktionen anders betrachtet werden sollten.

Die Entstehung dieser Konstruktionen, die ich nicht zu den identifizierenden Appositionen rechne, lässt sich auf übersetzungstechnische, übersetzerpsychologische Gründe zurückführen, die auf die in dieser Zeit, in der Periode der Übersetzungsliteratur, verbreitete, allgemein und üblich gewordene Übersetzer-Kopisten-Praxis zurückgehen.

3.2. Zusammenfassend lässt sich aufgrund der dargestellten Erscheinungen sagen, dass sich in der Addition solcher individueller Bestrebungen die Keime der Herausbildung der Literatursprache verbergen. In dieser Zeit entstand und stabilisierte sich - ein spezifischer Übersetzer-Kopisten-Stil, von dem wir behaupten können, dass er sich zu einem Stilideal erhob. Ja man kann sogar die Feststellung wagen, dass diese — aus dem Boden der Übersetzungsliteratur erblühte-Art der Textgestaltung und -verschönerung zu einer Stileigentümlichkeit der Schriftsprache jener Zeit wurde und später bereits auch selbständig, unabhängig von ihren Entstehungsumständen fungierte.

\section{Literatur}

Antal, László 1964. A magyar jelzô három különbözô nyelvtani koncepció fényében [Das ungarische Attribut im Lichte drei verschiedener grammatischer Konzeptionen]. In: Magyar Nyelv $60: 61-8$.

Balázs, János 1954. Sylvester János és a XVI. századi magyar irodalmi nyelv [János Sylvester und die ungarische Literatursprache des 16. Jh.]. In: Magyar Nyelv $50: 126-9$. 
Bárczi, Géza 1966. A magyar irodalmi nyelv kialakulása. [Die Entstehung der ungarischen Literatursprache] A Debreceni Kossuth Lajos Tudományegyetem Nyelvtudományi Intézetének Évkönyve. In: Magyar Nyelvjárások $12: 4-5$.

Benkő, Loránd 1960. A magyar irodalmi írásbeliség a felvilágosodás korának első szakaszában [Die ungarische literarische Schriftlichkeit im ersten Abschnitt der Aufklärung]. Akadémiai Kiadó, Budapest.

Benkő, Loránd 1980. Az Árpád-kor magyar nyelvû szövegemlékei [Ungarischsprachige Textdenkmäler der Árpádenzeit]. Akadémiai Kiadó, Budapest.

Benkő, Loránd 1988. A történeti nyelvtudomány alapjai [Die Grundlagen der historischen Sprachwissenschaft]. Tankönyvkiadó, Budapest.

Benkő, Loránd-Endre Rácz (Hg.) 1995. A magyar nyelv történeti nyelvtana 2/2: A kései ómagyar kor. Mondattan. Szöveggrammatika [Historische Grammatik des Ungarischen 2/2: Die spätaltungarische Zeit. Satzlehre. Textgrammatik]. Akadémiai Kiadó, Budapest.

Berrár, Jolán 1957. Magyar történeti mondattan [Ungarische historische Syntax]. Tankönyvkiadó, Budapest.

Berrár, Jolán 1967. A jelzős szerkezetek [Die attributiven Konstruktionen]. In: Géza Bárczi - Loránd Benkô - Jolán Berrár (Hg.) A magyar nyelv története [Die Geschichte der ungarischen Sprache], 450-5. Tankönyvkiadó, Budapest.

Farkas, Vilmos 1962. A többtagú (halmozott) mondatrészek [Die mehrgliedrigen (gehäuften) Satzteile]. In: Tompa (1962b, 315-27).

Hámori, Antónia S. 1981. Kötôszóhasználat az ómagyar kor értelmezôs szerkezeteiben [Konjunktionsgebrauch in den appositionalen Syntagmen der altungarischen Zeit]. In: Magyar Nyelv 78:148-51.

Hámori, Antónia S. 1991. Az értelmező jelzős szerkezetek [Die appositionalen Attributivsyntagmen]. In: Loránd Benkő- Erzsébet E. Abaffy - Endre Rácz (Hg.) A magyar nyelv történeti nyelvtana 1: A korai ómagyar kor és előzményei [Historische Grammatik des Ungarischen 1: Die frühaltungarische Zeit und ihre Vorereignisse], 706-11. Akadémiai Kiadó, Budapest.

Hámori, Antónia S. 1995. Az értelmező jelzős szerkezetek [Die appositionalen Attributivsyntagmen]. In: Benkő-Rácz (1995, 385-406).

Horváth, János 1944. A magyar irodalmi múveltség kezdetei Szent Istvántól Mohácsig [Anfänge der ungarischen literarischen Bildung vom hl. Stephan bis Mohács]. Magyar Szemle Társaság, Budapest.

Horváth, János 1980. A magyar irodalom fejlődéstörténete [Entwicklungsgeschichte der ungarischen Literatur]. Akadémiai Kiadó, Budapest.

Jakab, István 1977. Az értelmező és az értelmezett szószerkezeti viszonya [Das syntagmatische Verhältnis von Bezugswort und Apposition]. In: Magyar Nyelvốr 101:9-19.

Jakab, István 1978. Igen, az értelmezô mellérendelés [Ja, die Apposition ist Beiordnung]. In: Magyar Nyelvőr 102: 293-8. 
Juhász, Dezsô 1992. A kötôszók. A módosítószók [Die Konjunktionen. Die Modalwörter]. In: Loránd Benkő-Erzsébet E. Abaffy (Hg.) A magyar nyelv történeti nyelvtana 2/1: A kései ómagyar kor: morfematika [Historische Grammatik des Ungarischen 2/1: Die spätaltungarische Zeit: Morphematik], 772-814; 815-38. Akadémiai Kiadó, Budapest.

Kardosné Balogh, Judit 1989. A mellérendelő szószerkezetek és határeseteik [Die beiordnenden Syntagmen und ihre Grenzfälle]. In: Endre Rácz (Hg.) Fejezetek a magyar leíró nyelvtan köréből [Abschnitte aus dem Bereich der ungarischen deskriptiven Grammatik], 57-90. Tankönyvkiadó, Budapest.

Károly, Sándor 1958. Az értelmező és az értelmezői mondat a magyarban [Apposition und Appositionalsatz im Ungarischen]. Nyelvtudományi Értekezések 16. Akadémiai Kiadó, Budapest.

Károly, Sándor 1961. Az irodalmi nyelv, köznyelv, írott nyelv elnevezésekról [Über die Bezeichnungen Literatursprache, Umgangsprache, Schriftsprache]. In: Magyar Nyelvőr 85 : $385-98$.

Károly, Sándor 1978. Valóban mellérendelés az értelmező? [Ist die Apposition wirklich eine Beiordnung?]. In: Magyar Nyelvôr $102: 46-50$.

Mezey, László 1955. Irodalmi anyanyelvû́ségünk kezdetei az Árpád-kor végén [Anfänge unserer literarischen Muttersprachlichkeit am Ende der Árpádenzeit]. Akadémiai Kiadó, Budapest.

Nagy, Ilona 1981. A Jordánszky Kódex fordítástechnikai sajátságairól [Über die übersetzungtechnischen Spezifika des Jordánszky-Kodex]. In: Magyar Nyelv $77: 41-8$.

Pais, Dezső 1953. A magyar irodalmi nyelv [Die ungarische Literatursprache]. In: I. Osztály Közleményei IV., 425-66. Magyar Tudományos Akadémia, Budapest.

Papp, Zsuzsanna 1995. A mellérendelő szerkezetek. A mellérendelő mondatok. [Die beiordnenden Syntagmen. Die beiordnenden Sätze]. In: Benkő-Rácz (1995, 474-505; 719-57).

Papp, Zsuzsanna 1996. Értsed [Verstehe es]. In: János L. Nagy-Emma Rozgonyi (Hg.) Absztrakció és valóság. Békési Imre köszöntése [Abstraction und Wirklichkeit. Festschrift für Imre Békési], 237-41. JGYTF Kiadó, Szeged.

Pusztai, Ferenc 1978. A magyar irodalom és a magyar nyelv fejlödéstörténete [Ungarische Literatur und Entwicklungsgeschichte der ungarischen Sprache]. In: Magyar Nyelv 74: 385-90.

Pusztai, István 1991. Vitkovics-kódex 1525. A nyelvemlék hasonmása és betûhû́ átirata bevezetéssel és jegyzetekkel. [Vitkovics-Kodex. 1525. Facsimile und buchstabentreue Transkription des Sprachdenkmals mit Einleitung und Anmerkungen.]. Régi Magyar Kódexek 12. sz. Magyar Tudományos Akadémia Nyelvtudományi Intézete, Budapest.

Rácz, Endre 1963. A magyar nyelv következtető mondatainak története a XVI. század elejéig [Geschichte der Konklusivsätze der ungarischen Sprache bis zum Beginn des 16. Jh.]. In: Néprajz és Nyelvtudomány $7: 237-41$.

Simonyi, Zsigmond 1881. A magyar kötőszók [Die ungarischen Konjunktionen]. Magyar Tudományos Akadémia, Budapest. 
Szabó, Attila T. (Hg.) 1982. Erdélyi magyar szótörténeti tár III [Historisches Wörterbuch des siebenbürgisch-ungarischen Wortschatzes Bd. III]. Kriterion, Bukarest.

Szabó, Dénes 1958. A mai magyar nyelv [Die heutige ungarische Sprache]. Kézirat. Budapest.

Szarvas, Gábor-Zsigmond Simonyi 1890-1893. Magyar nyelvtörténeti szótár I-III [Sprachhistorisches Wörterbuch des Ungarischen I-III]. Hornyánszky Viktor Akadémiai Könyvkereskedése, Budapest.

Szathmári, István 1968. Régi nyelvtanaink és egységesülő irodalmi nyelvünk [Unsere alten Grammatiken und unsere sich vereinheitlichende Literatursprache]. Akadémiai Kiadó, Budapest.

Szathmári, István 1980. A magyar jelentéstan kezdetei [Anfänge der ungarischen Semantik]. In: Endre Rácz - István Szatmári (Hg.) Tanulmányok a mai magyar nyelv szókészlettana és jelentéstana köréből [Studien aus dem Bereich der Lexikologie und Semantik der heutigen ungarischen Sprache], 237-52. Tankönyvkiadó, Budapest.

Tompa, József 1962a. A jelző [Das Attribut]. In: Tompa (1962b, 260-313).

Tompa, József (Hg.) 1962b. A mai magyar nyelv rendszere II [System der heutigen ungarischen Sprache II]. Akadémiai Kiadó, Budapest.

Velcsov, Mártonné 1987-1988. Nyelvemlékeink nám szavának eredete [Die Etymologie von nám]. In: Néprajz és Nyelvtudomány 31-32:211-7.

Velcsov, Mártonné 1997. A nám kötôszó latin eredetéről [Über die lateinische Herkunft der Konjunktion nám]. In: László Büky (Hg.) Nyíri Antal kilencvenéves [Antal Nyíri ist 90 Jahre alt], 173-7. JATE, Szeged.

Adresse der Verfasserin: Zsuzsanna Papp

Institut für Sprachwissenschaft

der Ungarischen Akademie der Wissenschaften

Budapest

Benczúr utca 33

$\mathrm{H}-1068$

Ungarn

pappzs@nytud.hu 